\title{
Mathematical Approach to Determination of the Pressure at the Point of Leak in Natural Gas Pipeline
}

\author{
Ubanozie Julian Obibuike, Anthony Kerunwa, Mathew Udechukwu, Remmy Chindu Eluagu, \\ Anthony Chemazu Igbojionu, Stanley Toochukwu Ekwueme*
}

Department of Petroleum Engineering, Federal University of Technology, Owerri (FUTO), Nigeria

Email address:

stanleyekwueme@yahoo.com (S. T Ekwueme)

${ }^{*}$ Corresponding author

\section{To cite this article:}

Ubanozie Julian Obibuike, Anthony Kerunwa, Mathew Udechukwu, Remmy Chindu Eluagu, Anthony Chemazu Igbojionu, Stanley Toochukwu Ekwueme. Mathematical Approach to Determination of the Pressure at the Point of Leak in Natural Gas Pipeline. International Journal of Oil, Gas and Coal Engineering. Vol. 8, No. 1, 2020, pp. 22-27. doi: 10.11648/j.ogce.20200801.14

Received: December 30, 2019; Accepted: January 13, 2020; Published: February 10, 2020

\begin{abstract}
In this paper, a model is developed for the estimation of the pressure at the point of leak in a natural gas pipeline experiencing leak. The model is developed by comparing two equations developed for leak localization in natural gas pipeline corresponding to the upstream and the downstream sections of the pipeline respectively. The determination of the pressure at the point of leak which is the dominant parameter for the two models ensures the accuracy and equality of the two leak localization equation regardless of the section of the pipeline considered. The model for the point of leak develops helps to monitor pipeline pressure profile for the leak and the no leak case and helps to detect the exact point of leak in the pipeline depicted by the pressure reductions gotten by comparing the pressure profile of the no leak and the leaking pipeline cases. In the work three cases were considered. In each of the cases, the pressure at the point of leak were estimate with the corresponding location where the leak occurred. It is also realized as expected that the calculated pressures at the point of leak were lower than the expected pressures at the same point if the pipeline were not subject to leak. The results gotten show that the pressure at the point of leak for the three cases are 738 psi, 678.33 psi and 933.89 psi for case 1 , case 2 and case 3 respectively. These pressure values occurred at locations 139.5 miles, 86.48 miles and 34.94 miles from the inlet point for case 1 , case 2 and case 3 respectively.
\end{abstract}

Keywords: Leak Pressure, Natural Gas, Pressure Profile, Mathematical Modeling, Point of Leak

\section{Introduction}

Hydrocarbon fluid flow is essential in oil and gas operations as fluid movements at various stages of operations are inevitably encountered. It becomes necessary to adequately study and understand the mechanisms of fluid flow in hydrocarbon pipelines. Oftentimes, owing to complexities and factors either environmentally or human induced, disturbances are created along the pipeline of flow and thus leaks are encountered in the pipelines [1]. Aside the economic consequences in terms of huge financial losses suffered by the government and the operating companies, leak of hydrocarbon fluids poses great environmental consequences to host communities and has been noted for varied unfavourable impacts to the underlying ecosystem [2].
However, care should be taken to understand that although complete eradication of leaks in pipeline may be viewed as 'wishful' but calculable efforts must be made to reduce the occurrences of leak and/or the magnitude of damage to environment resulting from effluent fluid in leak cases [3, 4]. One way of achieving this is the careful monitoring and supervision of fluid pipeline networks and the use of effective and efficient software based leak detection systems to improve alertness and quicker response procedures. In leak detection methodology, the contention is not to safeguard the pipeline against leak but to reduce severity of leak by an efficient management of leak occurrence.

Two broad classifications exit for leak detection systems; these are externally (released fluid detection) and internally (observing hydraulic behavior) based methods. The externally based methods are called physical inspection 
methods and are done to estimate the location and size of leaks [5, 6]. Physical inspection comprise fluid sampling; soil monitoring; flow-rate monitoring; and acoustic-, optical-, and satellite-based hyperspectral imaging. In most cases, the physical inspection leads in accurate determination of the location of leak and also size of leak. But this comes with additional cost of production shutdown including high cost and long time to run the physical detection, which is a very essential factor when considering pipelines of long distances [7-9].

The internal methods fall into a broad category referred to as model based leak detection methods. These models are mathematical equations analytically or numerically developed to address leak phenomena in pipelines. The Mathematical-modeling approach helps to detect oil leak by solving mass-conservation, momentum-conservation, and energy- balance equations which constitute the governing equations, thus leading to a quick evaluation at a low expense $[10,11]$. One advantage of this method is that the pipeline is monitored continuously without any interference to pipeline operations. One disadvantage of this method is the necessary provision of flow parametres which may not always be readily available. And also mathematically modelled leak detection methods may have higher uncertainties than that from physical inspection methods [12, 13].

As has been stated by Obibuike et al, [14], Leak localization is one of the greatest parametres to be calculated during leak incidence. Ability to localize leak leads to swift response on the pipeline system leading to reduction in material and financial losses.

Obibuike et al [15] provided equations to locate leak in natural gas pipeline. In the development of the model, they took cognizance of two sections of the pipeline, the upstream and the downstream section respectively. Leak models were developed for each section of the pipeline. This resulted to two analytical equations being developed for leak location. Solving the two equations require the knowledge of the common pressure parameter called the pressure at the point of leak.

Since the pressure at the point of leak is crucial and sequel to the accurately determination of the leak location albeit through mathematical means, it is therefore imperative that a means be developed for the accurate determination of this parameter. In this work, we have used mathematical approach to determine the pressure at the point of leak using two leak location equations developed for the upstream and downstream sections of the pipeline respectively. The idea in the development of the model is that since the leak distances calculated using the leak equations from either sections of the pipeline are equal, then the pressure at the point of leak which is a common variable in both sides of the equation can be determined by equating the mathematical models from the upstream and downstream sections together and solving the resulting equation extensively.

The pressure at the point of leak will enable the estimation of the fluid loss volume from the leak opening and this will help in estimation of the financial loss due to leak incidence in a natural gas pipeline subject to leak.

\section{Methods}

Obibuike et al (2019) developed the equation for leak location in natural gas pipeline. The equations were developed both for the upstream and downstream sections of the pipeline respectively. The upstream leak location was given as $\mathrm{X}_{1}$ while the downstream leak location was given as $\mathrm{X}_{2}$.

For the upstream section of the pipeline, the equation for leak location was given as

$$
X_{1}=L\left[\left(\frac{Q_{f}}{q}\right)^{2}\left(\frac{P_{1}{ }^{2}-P_{f}{ }^{2}}{P_{1}{ }^{2}-P_{2}^{2}}\right)\right]
$$

The leak location equation for the downstream section was also given as

$$
X_{2}=L\left\{1-\left[\left(\frac{q}{Q_{R}}\right)^{2} \frac{\left(P_{f}^{2}-P_{R}^{2}\right)}{\left(P_{1}^{2}-P_{2}^{2}\right)}\right]\right\}
$$

Where

$\mathrm{X}_{1}, \mathrm{X}_{2}$ are the leak location for the upstream and downstream section respectively, miles

$\mathrm{q}=$ gas flowrate in $\mathrm{ft}^{3} / \mathrm{hr}$,

$\mathrm{P}_{1}=$ inlet pressure in no leak, psia,

$\mathrm{P}_{2}=$ outlet pressure in no leak, psia,

$L=$ Length of the pipeline, miles

$\mathrm{q}=$ gas flowrate in no leak $\mathrm{ft}^{3} / \mathrm{hr}$

$\mathrm{Q}_{1}=$ the inlet gas flowrate of the no leak case in $\mathrm{ft}^{3} / \mathrm{hr}$

$\mathrm{Q}_{2}=$ the output gas flowrate of the no leak case in $\mathrm{ft}^{3} / \mathrm{hr}$

$Q_{f}=$ the flowrate at the point where leak has occurred $\mathrm{ft}^{3} / \mathrm{hr}$

$P_{f}=$ the pressure midstream of pipeline at the instant just before leak occurs

$Q_{R}=$ the recorded flowrate at the output section of the pipeline in $\mathrm{ft}^{3} / \mathrm{hr}$

$P_{R}=$ the recorded output pressure in psi

Regardless of the section of the pipeline chosen for analyses, the leak location is the same, thus

Leak location from upstream section=Leak location from downstream section

$$
\mathrm{X}_{1}=\mathrm{X}_{2}
$$

\subsection{Determination of Pressure at the Point of Leak}

The pressure at the point of leak can be determine from the leak location equations developed as given in equation 1 and equation 2 respectively. This pressure can be calculated by equating the two equations determined for the leak location by considering the upstream and downstream sections of the pipeline.

$$
\boldsymbol{L}\left[\left(\frac{Q_{f}}{q}\right)^{2}\left(\frac{P_{1}{ }^{2}-P_{f}{ }^{2}}{{P_{1}}^{2}-P_{2}{ }^{2}}\right)\right]=L\left\{1-\left[\left(\frac{q}{Q_{R}}\right)^{2} \frac{\left(P_{f}^{2}-P_{R}{ }^{2}\right)}{\left(P_{1}{ }^{2}-P_{2}{ }^{2}\right)}\right]\right\}
$$

But $Q_{F}=Q_{R}$ (flow is unaltered in the downstream section of the pipeline) 


$$
\begin{gathered}
\left(\frac{Q_{R}}{q}\right)^{2}\left(P_{1}^{2}-P_{f}^{2}\right)=\left(P_{1}^{2}-P_{2}^{2}\right)-\left(\frac{q}{Q_{R}}\right)^{2}\left(P_{f}^{2}-P_{R}^{2}\right) \\
\left(\frac{Q_{R}}{q}\right)^{2}\left(P_{1}^{2}-P_{f}^{2}\right)+\left(\frac{q}{Q_{R}}\right)^{2}\left(P_{f}^{2}-P_{R}^{2}\right)=\left(P_{1}^{2}-P_{2}{ }^{2}\right) \\
\left(\frac{Q_{R} P_{1}}{q}\right)^{2}-\left(\frac{Q_{R} P_{f}}{q}\right)^{2}+\left(\frac{q P_{f}}{Q_{R}}\right)^{2}-\left(\frac{q P_{R}}{Q_{R}}\right)^{2}=\left(P_{1}{ }^{2}-P_{2}{ }^{2}\right) \\
P_{f}{ }^{2}\left[\left(\frac{q}{Q_{R}}\right)^{2}-\left(\frac{Q_{R}}{q}\right)^{2}\right]=\left(P_{1}{ }^{2}-P_{2}{ }^{2}\right)-\left(\frac{Q_{R} P_{1}}{q}\right)^{2}+\left(\frac{q P_{R}}{Q_{R}}\right)^{2} \\
P_{f}=\left[\frac{\left(P_{1}{ }^{2}-P_{2}{ }^{2}\right)-\left(\frac{Q_{R} P_{1}}{q}\right)^{2}+\left(\frac{q P_{R}}{Q_{R}}\right)^{2}}{\left(\frac{q}{Q_{R}}\right)^{2}-\left(\frac{Q_{R}}{q}\right)^{2}}\right]^{0.5}
\end{gathered}
$$

Equation 11 is the equation for the pressure at the point of leak in the natural gas pipeline subject to leak occurrence

\subsection{Pressure Gradient Equation}

It is necessary also to determine the pressure gradient for the natural gas pipeline. This will enable the determination of pressure profile both in the absence and in the presence of leak.

From Weymouth's equation for fluid flow in horizontal pipeline

$$
q=3.23 \frac{T_{b}}{P_{b}} \sqrt{\frac{1}{f}} \sqrt{\frac{\left(P_{1}^{2}-P_{2}^{2}\right) D^{5}}{\gamma \bar{T} \bar{z} L}}
$$

Weymouth gave a relation for the friction factor as

$$
f=\frac{0.032}{D^{1 / 3}}
$$

Where $\mathrm{D}$ is the pipe diameter in inches

Putting equation 13 into equ 12 the Weymouth's equation simplifies to

$$
q=18.06 \frac{T_{b}}{P_{b}} \sqrt{\frac{\left(P_{1}{ }^{2}-P_{2}{ }^{2}\right) D^{16 / 3}}{\gamma \bar{T} \bar{Z} L}}
$$

To calculate the pressure gradient, this is the pressure at every section of the pipeline from inlet to outlet, we make the pressure term the subject of the formula. Bear in mind that this is a steady state system, thus the gas compressibility factor and temperature is uniform at every section of the pipeline throughout the flow period.

The pressure drawdown from inlet to outlet of the pipeline is given by

$$
\left(P_{1}^{2}-P_{2}^{2}\right)=\left(\frac{q P_{b}}{18.062 T_{b}}\right)^{2} \frac{\gamma \bar{T} \bar{z} L}{D^{16 / 3}}
$$

Thus the pressure gradient at point $\mathrm{x}$ from the inlet is given by

$$
\frac{\left(P_{1}^{2}-P_{x}^{2}\right)}{L}=\left(\frac{q P_{b}}{18.062 T_{b}}\right)^{2} \frac{\gamma \bar{T} \bar{z}}{D^{16 / 3}}
$$

Thus the pressure at point $\mathrm{x}$ at all section of the pipeline is given by

$$
P_{x}^{2}=P_{1}^{2}-\left[\left(\frac{q P_{b}}{18.062 T_{b}}\right)^{2} \frac{\gamma \bar{T} \bar{z}}{D^{\frac{16}{3}}}\right] * L
$$

Thus

$$
P_{x}=\sqrt{P_{1}{ }^{2}-\left[\left(\frac{q P_{b}}{18.062 T_{b}}\right)^{2} \frac{\gamma \bar{T} \bar{z}}{D^{\frac{16}{3}}}\right] * L}
$$

If we denote

$$
P_{G}=\left(\frac{q P_{b}}{18.062 T_{b}}\right)^{2} \frac{\gamma \bar{T} \bar{z}}{D^{16 / 3}}
$$

Then

$$
P_{x}=\sqrt{P_{1}^{2}-P_{G} * L}
$$

Where $\mathrm{P}_{\mathrm{G}}=$ pipeline gradient $\mathrm{psi} / \mathrm{mile}$

\subsection{Model Simulation}

The sequence in the simulation of the model are; Data gathering, Data validation, Simulation with Matlab software, Presentation of results. The data was collected from XYZ Gas Company operating in the Niger Delta of Nigeria. The data was validated with Matlab to ensure its correctness and compatibility with the pipeline system chosen. Matlab software was used to run the simulation to determine the leak location.

Table 1. Presentation of Input data.

\begin{tabular}{llll}
\hline \multirow{2}{*}{ Parameters } & Values & & \\
\cline { 2 - 4 } & Case 1 & Case 2 & Case 3 \\
\hline Pipeline length (miles) & 160 & 120 & 150 \\
Pipeline diameter (in.) & 15.5 & 12.09 & 14 \\
Inlet pressure (psia) & 1250 & 1000 & 1080 \\
Outlet pressure (psia) & 800 & 720 & 680 \\
Flowing fluid temp. $\left({ }^{\circ} \mathrm{F}\right)$ & 80 & 80 & 100 \\
Base temperature. $\left({ }^{\circ} \mathrm{F}\right)$ & 60 & 60 & 60 \\
Gas deviation factor & 0.85 & 0.88 & 0.95 \\
Gas specific gravity & 0.6 & 0.67 & 0.65 \\
Gas flowrate at inlet (MMscf/hr) & 4.365 & 1.746 & 2.679 \\
Output flowrate during leak (MMscf/hr) & 3.88 & 1.4 & 2 \\
Atmospheric pressure (psia) & 14.7 & 14.7 & 14.7 \\
Temperature at standard conditions $\left({ }^{\circ} \mathrm{R}\right)$ & 520 & 520 & 520 \\
Output pressure during leak (psia) & 671.73 & 678.33 & 755.78 \\
\hline
\end{tabular}

\section{Result and Discussions}

The results given in table 2 below show the pressure at the point of leak calculated from each leak location for each of the three cases considered.

\subsection{Results for Pressure at the Point of Leak for the Leak Locations Determined}

Table 2 below gives the results for the pressure at the point of leak for leak locations determined in each of the cases considered. 
Table 2. Table showing Results for the pressure at the point of leak.

\begin{tabular}{llll}
\hline Case & Leak Location (Miles) & Expected Pressure of the pipeline at leak point, psi & Pressure In Pipeline at Leak Point (Pf), psi \\
\hline case 1 & 139.5 & 871 & 738 \\
case 2 & 86.48 & 808.2 & 678.33 \\
case 3 & 34.94 & 1001.3 & 933.89 \\
\hline
\end{tabular}

From table 2, the pressure at the leak point is 738 psi for case 1, 678.33 psi for case 2 and 933.89 psi for case 3 . Correspondingly, the leak occurred at locations 139.5 miles, 86.48 miles and 34.94 miles from the inlet point for case 1, case 2 and case 3 respectively.

\subsection{Pressure Profile Graphs for the Three Cases}

For each of the following cases, the pressure profile for the leak and no leak case are given in graphical form.

The pipeline has a normal pressure profile signature given by the blue line in the absence of leak. This profile is intrinsic of the pipeline transporting fluid in steady state. When leak occurs, a perturbation is created which is felt by the pipeline. The pipeline responds by changing its pressure of flow. The red line depicts the pressure profile for the pipeline during leak. The red line as noticed from the figures below deviated from the blue line at the point of leak. Table 2 gives the pressures in the pipeline at the point of leak. These pressures differ from the pressures calculated for the pipeline at the same location where leak occurred for the same pipeline system that is not experiencing leak.

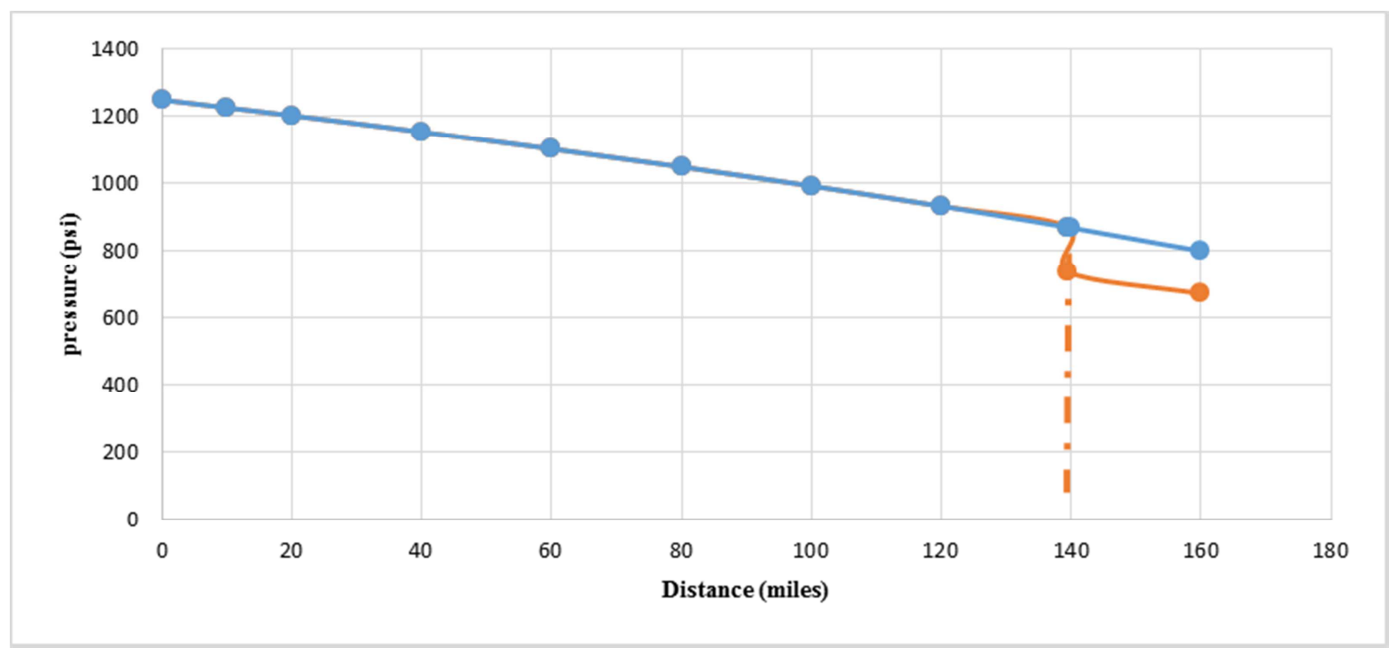

Figure 1. Pressure profile chart showing leak and no leak situations for case 1.

Figure 1 shows the pressure profile graph for case 1 . The leak occurred at 139.5 miles from the inlet point. The estimated pressure for the normal pipeline system if there is no leak at $139.5 \mathrm{~m}$ miles is $871 \mathrm{psi}$. Due to leak, the pressure at the leak location i.e. 139.5 miles from the inlet of the pipeline is calculated to be 738 psi. Thus there is a reduction in pressure of 133 psi due to leak. From the diagram notice that the blue line and the red line did not meet or cross at any point before the outlet of the pipeline. Thus the pressure profile for the leak case did not stabilize with the no leak case before the exit of the pipeline is reached.

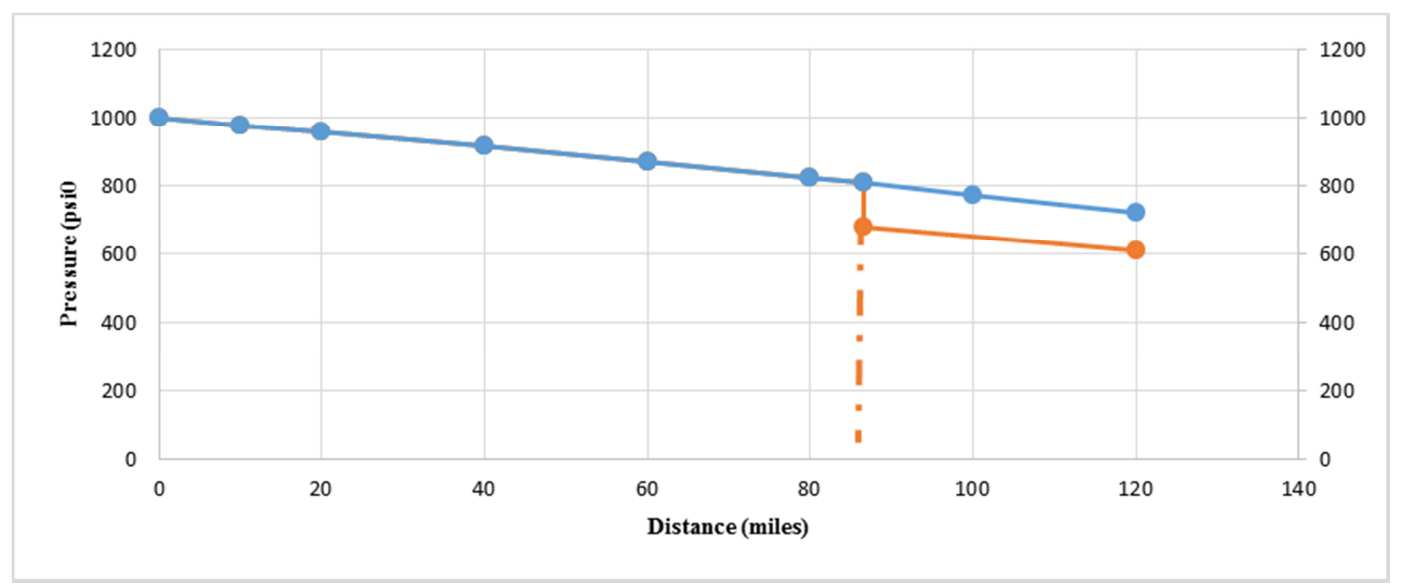

Figure 2. Pressure profile chart showing leak and no leak situations for case 2. 
From figure 2, the leak occurred at a distance of 86.48 miles from the inlet of the pipeline. The pressure of the pipeline experiencing leak just at the leak location is calculated to be 678.33 psi as given in table 2. The Expected pressure at that point according to the pressure profile for the pipeline if no leak has occurred is 808.2 psi. Thus, there is a pressure reduction of 129.87 psi due to leak at the point of leak considered. Also from the figure 2, it will be seen that the two pressure profile graphs did not meet or cross before the exit of the pipeline is reached which signifies that the pressure profile created by the leak incidence did not stabilize before the exit of the pipeline is reached.

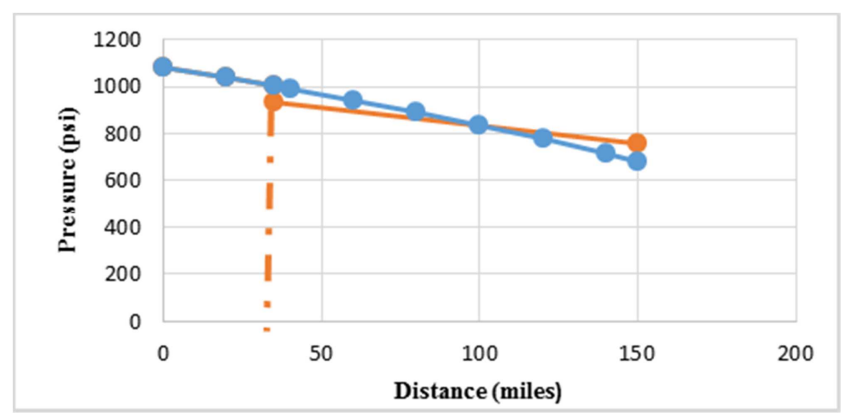

Figure 3. Pressure profile chart showing leak and no leak situations for case 3.

From figure 3, the leak occurred at a distance 34.94 miles from the inlet of the pipeline. The pressure in the leaking pipeline at the leak point is calculated to be 933.89 psi while the normal pressure for that same location if the pipeline were not subject to leak is 1001.3 psi. The leak occurrence caused a pressure reduction of 67.41 psi. Notice that from figure 3 , the red line depicting the pressure profile for the leak case meet with the blue line depicting the pressure profile for the no leak case at a distance of 100 miles from the pipeline and eventually crossed it. Thus the pressure profile for the leak case stabilized at 100 miles. The question that comes to mind is "what is the significance of the pressure profile overlap" as seen in the red line meeting and crossing the blue line".

\subsection{Explanation for the Pressure Profile Overlap}

Leak occurrence causes abrupt pressure reduction at the point of leak giving the pipeline a new pipeline hydraulic profile depicted by the red line in the figures above, which emanates at the point of leak towards the exit of the pipeline. Although this new pipeline profile is different from the normal pipeline pressure profile for a normal pipeline experiencing no leak. This deviations is caused by environmental perturbances created due to leak which has mad the pipeline open to external environmental influenced making the pipeline to function as an open system. Thus the downstream of the pipeline experiences pressure anomalies which tries to adjust to the normal pipeline profile depending on the nature of the leak and the type of fluid transported.
The pressure in the pipeline from the leak point to the pipeline outlet begins to build instead of the normal drawdown. This pressure build up is as a result of environmental forces on the pipeline in terms of fluid vortices which act at the pipeline leak location and travels downstream along the fluid stream to the outlet section of the pipeline. This environmental perturbation results in the pipeline pressure build-up. The pipeline pressure continues to build with time until a point where the red line intersects with the blue line. This point is called the critical pipeline pressure after leak. If at this point no remedial work has been done on the pipeline to curtail this pressure build up then the pressure will increase further from the intersect point and may ultimately lead to burst of the pipeline because the pressure head in the pipeline has risen beyond the burst pressure of the pipeline. Pipeline burst after leak can occur anywhere after intersect of the blue line with the red line.

Chemically, the reason for the pressure build up and ultimate burst of the pipeline after leak can be understood in terms of the reaction between the natural gas and the environmental air. When leak occurs, the natural gas in the pipeline is open to external force subject to air particle interference. A reactive fuel-air cloud forms that gives rise to chemically unstable compounds in the regions of the pipeline system leading to pressure buildup which when not controlled leads to burst of the pipeline.

\section{Conclusion}

Models for determination of pressure at the point of leak in a natural gas pipeline experiencing leak has been developed. Pipelines respond to leak by variation in pressures given by the pressure profile. Perturbances created by leak due to external influence on the pipeline system results in varied pressure profile in the pipeline for the normal pipeline and for the pipeline that is subject to leak. The pressure at the point of leak has been an interesting parameter to researchers, its determination will not only help to localize leak but also help to estimate the fluid flow velocity and flowrate from the leak opening in real-time.

From the results, the pressures at the point of leak for the three cases were determined. Also, the pressure expected for that point where leak occurred for a normal pipeline system experiencing no leak was given. As expected, in all the cases, the pressure at the point of leak for a leaking pipeline calculated were all lower than the expected pressure vales at the same location if the pipeline were not experiencing leak.

The model is useful in pipeline leak detection and overall pipeline monitoring and supervision especially in SCADA systems. The model is highly recommended to be used in any gas transportation system for efficient and reliable gas delivery operations. 


\section{References}

[1] Ekwueme, S. T (2014). Model for pipeline leak detection in natural gas pipeline. Paper presented at the annual STCE paper presentation. Warri, Delta state.

[2] Ekwueme, S. T (2014). A new mathematical model for pipeline leak detection in natural gas pipeline. Project submitted to Petroleum engineering Dept. FUTO.

[3] Hanmer, G., Mora, V., Fábio C. G., Sergio L. (2018). Modelling of Rapid Transients in Natural Gas Pipelines. Paper prepared for presentation at the PSIG Annual Meeting held in Deer Valley, Utah.

[4] Nicholas, E., Carpenter, P., Henrie, M., Hung, D., Kundert, C. (2017). A New Approach to Testing Performance of a Pipeline Leak Detection System. Paper prepared for presentation at the PSIG Annual Meeting held in Atlanta, Georgia, USA.

[5] Hauge, E., Aamo, O. M., and Godhavn, J.-M. (2009): ModelBased Monitoring and Leak Detection in Oil and Gas Pipelines. SPE Proj Fac \& Const 4 (3): 53-60. SPE-114218PA.

[6] Jin Mingang (2019). Investigation on Parameters Affecting the Performance of Negative Pressure Wave Leak Detection Systems. Paper prepared for presentation at the PSIG Annual Meeting held in London, England.

[7] Balda Rivas, K. V. and Civan, F. (2013): Application of Mass Balance and Transient Flow Modeling for Leak Detection in Liquid Pipelines. Presented at the SPE Production and Operations Symposium, Oklahoma City, Oklahoma, USA, 23-26 March. SPE-164520-MS.

[8] Elliott, J., Fletcher, R., and Wrigglesworth, M. (2008):
Seeking the Hidden Threat: Applications of a New Approach in Pipeline Leak Detection. Presented at the Abu Dhabi International Petroleum Exhibition and Conference, Abu Dhabi, 3-6 November. SPE-118070-MS.

[9] Zhang, J., Hoffman, A., Murphy, K., Lewis, J., Twomey, M. (1996). Review of pipeline leak detection technologies. Pipeline Simulation Interest Group.

[10] Boaz, L., Kaijage, S. and Sinde, R. (2014). An overview of pipeline leak detection and location systems. Pan African International Conference on Information Science, Computing and Telecommunications, (pp. 133-136).

[11] Ferrante, M., Brunone, B. and Meniconi, S. (2007). Wavelets for the analysis of transient pressure signals for leak detection. ASCE 133 (11), (pp. 1274-1282).

[12] Gajbhiye, R. N. and Kam, S. I. (2008). Leak Detection in Subsea Pipeline: A Mechanistic Modeling Approach with Fixed Pressure Boundaries. Presented at the Offshore Technology Conference, Houston, 5-8 May. OTC-19347-MS.

[13] Ghazali, M. F. (2012). Leak detection using instantaneous frequency analysis (PhD thesis), University of Sheffield, United Kingdom.

[14] Obibuike Ubanozie Julian, Ekwueme Stanley Toochukwu, Ohia Nnaemeka Princewill, Igwilo Kevin Chinwuba, Onyejekwe Ifeanyi Michael, Igbojionu Anthony Chemazu. (2019). Analytical Model for the Estimation of Leak Location in Natural Gas Pipeline. International Journal of Oil, Gas and Coal Engineering. Vol. $\mathrm{x}, \quad$ No. $\mathrm{x}, \mathrm{pp} . \quad \mathrm{x}-\mathrm{x} . \quad$ doi: 10.11648/j.xxx.xxxxxxxx.xx.

[15] Obibuike, U. J. (2019). Real Time Estimation of Leak Location in a Natural Gas Pipeline - A Fluid Flow Modeling Approach. Ph. D Thesis submitted to Postgraduate school, FUTO, Owerri, Nigeria. 